\title{
Precision measurements of the Top quark with the ATLAS experiment
}

\author{
Susana Amor dos Santos*i \\ LIP, Departamento de Física, Universidade de Coimbra P-3004-516 Coimbra, Portugal \\ E-mail: samorecern.ch
}

\begin{abstract}
The top quark is the heaviest known fundamental particle. As it is the only quark that decays before it hadronizes, this allows the unique opportunity to probe the properties of bare quarks. In this paper the most recent precision measurements of the top quark properties, performed by the ATLAS experiment using data from proton-proton collisions at $\sqrt{s}=8 \mathrm{TeV}$ and $\sqrt{s}=$ $7 \mathrm{TeV}$ are reviewed. The top pair and single top production cross-sections, including differential distributions, alongside some top quark properties measurements are shown. Interpretations in terms of limits on physics beyond the standard model and searches for new physics using top quarks will also be presented.
\end{abstract}

XIIth International Conference on Heavy Quarks \& Leptons 2014,

25-29 August 2014

Schloss Waldthausen, Mainz, Germany

\footnotetext{
*Speaker.

${ }^{\dagger}$ On behalf of the ATLAS Collaboration
} 


\section{Introduction}

Discovered almost 20 years ago by the Tevatron experiments [1,2], the top quark is the heaviest fundamental particle $\left(m_{t}=173.34 \pm 0.27\right.$ (stat.) \pm 0.71 (syst.) $\mathrm{GeV}$ [3]) known so far. With a charge of $+2 / 3|e|$ ( $e$ is the electron charge) and spin $1 / 2$, the top quark completes the 3 family structure of the Standard Model (SM). The top quark decays mainly into a $W$ boson and a $b$ quark, with a branching ratio of $\sim 1$ according to the SM. The branching ratios for the other electroweak decays, into an $s$ quark $\operatorname{Br}(t \rightarrow s W) \leq 0.18 \%$ or a $d$ quark $\operatorname{Br}(t \rightarrow d W) \leq 0.02 \%$, are diminut when compared with the dominant channel [4]. One of the striking features of this quark is the value of its mass, implying a large coupling to the SM higgs boson, which can be studied in processes such as associated production of top pairs with a higgs boson. Since its mass value is so distinctive from other fermions, this may suggest the top quark plays a more fundamental role in the eletroweak symmetry breaking mechanism. Furthermore, the study of the top quark mass allows for consistency checks of the SM, as will be detailed bellow. The lifetime, at least one order of magnitude smaller than the typical hadronization time scale $O\left(10^{-23}\right) \mathrm{s}$, was measured to be $\tau_{t}=\left(3.29_{-0.67}^{+0.90}\right) \times 10^{-25} \mathrm{~s}$ [5]. This implies top quarks decay before hadronization occurs, allowing the reconstruction of bare quarks and propagation of the spin information to the final state decay products. Once again, this is an unique property among all the quarks. The top quark events are significant background to other studies with similar final topologies and possibly smaller production cross-sections, such as the higgs boson analysis. Therefore, the better understanding of the top quark properties, may lead to better results in these analyses as well. Ultimately, due to its unique properties, the top quark may have enhanced couplings to many new particles, which may hint for new physics beyond the SM.

There are two main mechanisms of producing top quarks: through strong interactions creating a pair of top quarks or through electroweak processes obtaining only a single top quark or antiquark. At the LHC, top quarks are mainly produced in pairs through gluon fusion or quark anti-quark annihilation with a predicted cross-section of $177.3 \pm 9.0_{-6.0}^{+4.6} \mathrm{pb}$ at $7 \mathrm{TeV}$ and $252.9 \pm 11.7_{-8.6}^{+6.4} \mathrm{pb}$ at $8 \mathrm{TeV}$, both calculated at next-to-next-to leading order (NNLO) with next-to-next-to leading logarithmic (NNLL) corrections [6-10]. Nonetheless a significant number of top quarks are produced through electroweak processes and can be classified into three different channels according to the feynman diagrams: the $t$-channel $\left(g q^{\prime} \rightarrow q t \bar{b}\right)$, the $W t$ associated production $(b g \rightarrow W t)$ and the $s$-channel $\left(q \bar{q}^{\prime} \rightarrow t \bar{b}\right)$ of single top production. For proton-proton collisions at $\sqrt{s}=8 \mathrm{TeV}$ the production cross-sections are, respectively, 87.7 $7_{-1.9}^{+3.4} \mathrm{pb}$ [11] (NNLO+soft gluon corrections), $22.4 \pm 1.5 \mathrm{pb}$ [12] (approx. NNLO) and $5.6 \pm 0.2 \mathrm{pb}$ [13] (NNLL resummation). The production cross-sections at $\sqrt{s}=7 \mathrm{TeV}$ can also be found in these references.

In this paper, the experimental status of the measurements of the $V_{t b}$ coupling are reviewed, as well as measurements sensitive to gauge bosons couplings $(\gamma, \mathrm{W}, \mathrm{Z}$ and $\mathrm{H})$, which are particularly difficult measurements.

In order to measure the top quark properties, different analysis are performed for each production mechanism, according to their final state topology. In the $t \bar{t}$ pair production, the decays of the $W$ boson (to a quark and an anti-quark, or to a lepton and a neutrino), will define the final states: hadronic, single lepton, or dileptonic channels. The single lepton and dilepton channels are characterized by one charged lepton and at least four jets, or two charged leptons and at least two 
jets, respectively, and missing transverse energy (associated with the undetected neutrinos). This accounts for $\sim 35 \%$ of the $t \bar{t}$ production. The hadronic channel is the most probable one, with both $W$ bosons decaying into a pair of jets, however it is also the most difficult analysis to perform due to the major hadronic backgrounds. The $t$-channel of single top quark production is characterized by events with exactly one isolated lepton ( $e$ or $\mu$ ), missing transverse energy and two or three jets, where at least one is $b$-tagged. For the $W t$-channel event signature only the dileptonic final states are considered, thus requiring two isolated leptons of opposite electric charge, significant missing transverse momentum and one $b$-tagged central jet. In this paper the most recent results of the $t \bar{t}$ dilepton and single lepton analysis will be shown, as well as a summary of the single top quark analysis in all three channels using both at 7 and $8 \mathrm{TeV}$ data collected with the ATLAS experiment [14].

\section{Precision measurements of top quark properties}

\subsection{The $t \bar{t}$ production cross-section}

The ATLAS experiment has performed several measurements of the top quark pair production cross-section $\sigma_{t \bar{t}}$ using data from proton-proton collisions at $\sqrt{s}=7 \mathrm{TeV}$ and $\sqrt{s}=8 \mathrm{TeV}$, corresponding to an integrated luminosity of $4.6 \mathrm{fb}^{-1}$ and $20.3 \mathrm{fb}^{-1}$, respectively.

In the dilepton topology, the inclusive $t \bar{t}$ production cross-section $\sigma_{t \bar{t}}$ has been measured, using $t \bar{t}$ events with an opposite-charge $e \mu$ pair in the final state. The $\sigma_{t \bar{t}}$ was measured with $\sim 4 \%$ uncertainty as $\sigma_{t \bar{t}}(8 \mathrm{TeV})=242.4 \pm 1.7$ (stat.) \pm 5.5 (syst.) \pm 7.5 (lumi.) \pm 4.2 (beamen.) pb and $\sigma_{t \bar{t}}(7 \mathrm{TeV})=182.9 \pm 3.1$ (stat.) \pm 4.2 (syst.) \pm 3.6 (lumi.) \pm 3.2 (beamen.) pb [15]. These results are consistent with recent theoretical QCD calculations at NNLO and within its uncertainty band [6-10].

For the semileptonic topology, the ATLAS analysis was able to provide differential crosssections already at $\sqrt{s}=7 \mathrm{TeV}$, which represent precision tests of current theoretical predictions.

The fiducial differential cross-sections were studied as a function of the jet multiplicity for up to eight jets, using jet transverse momentum $\left(p_{\mathrm{T}}\right)$ thresholds of $25,40,60$, and $80 \mathrm{GeV}$, and as a function of jet transverse momentum up to the fifth jet [16]. In Figure 1 some of the obtained results are shown, after background subtraction and corrections for all detector effects, within a kinematic range closely matched to the experimental acceptance. Several QCD-based Monte Carlo (MC) models were compared with the data results, which provided discriminating power amongst the MC model predictions. Sensitivity to the parton shower modelling was found at the higher jet multiplicities, at high transverse momentum of the leading jet and in the transverse momentum spectrum of the fifth leading jet. The MC@NLO+HERWIG MC simulation was found to predict too few events at higher jet multiplicities when comparing with data events. Also, MC@NLO is disfavoured by data in the additional jet $p_{\mathrm{T}}$ distributions, which are too soft at high transverse momenta. In contrast, predictions from POWHEG showered with PYTHIA are consistent with the data within the total uncertainties of the measurements. This agreement can be further improved by limiting the hard radiations in POWHEG using free model parameters. The comparison to different $\alpha_{S}$ settings using the ALPGEN+PYTHIA sample indicates that the data prefer a softer parton-shower, i.e. a smaller value of $\alpha_{S}$. 


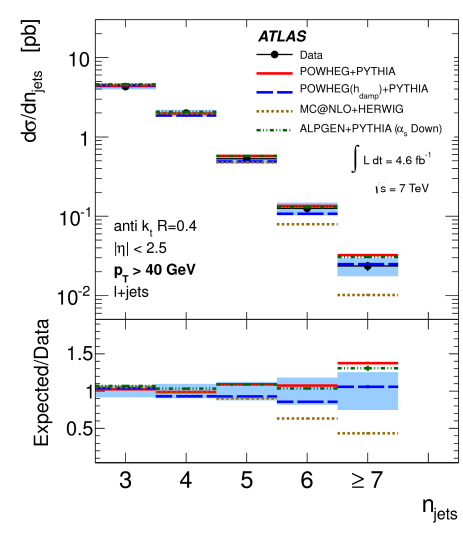

(a)

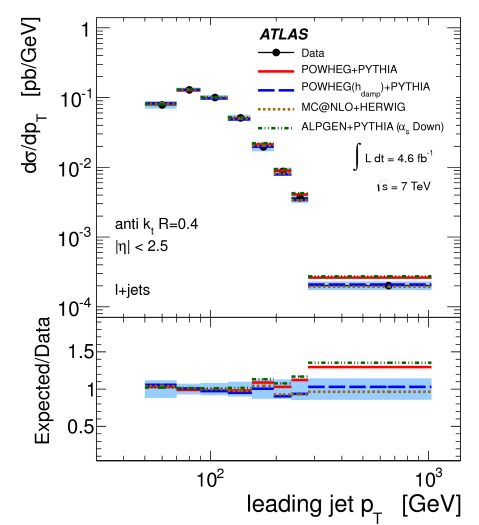

(b)

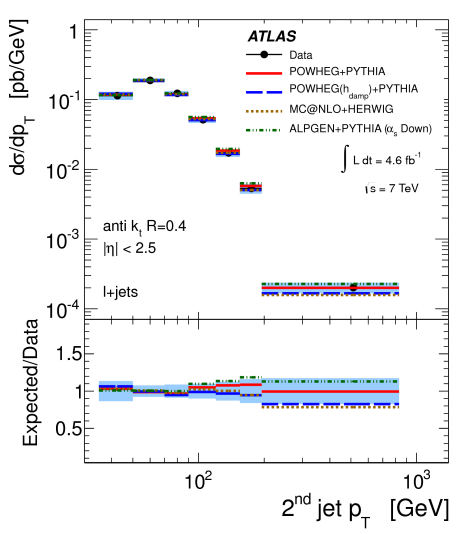

(c)

Figure 1: The $t \bar{t}$ cross-section for the average of the $e$ and $\mu$ channels as a function of the jet multiplicity (a) for the jet $p_{T}$ threshold of $40 \mathrm{GeV}$ and as a function of the jet $p_{\mathrm{T}}$ for the (b) leading and (c) 2nd jet. For more detailed information see [16].

The study of the normalised differential cross-sections for $t \bar{t}$ as a function of the top-quark $p_{\mathrm{T}}$, and of the mass, $p_{\mathrm{T}}$, and rapidity of the $t \bar{t}$ system in the single lepton channel [17] was also performed. The measured spectra are corrected for detector efficiency and resolution effects and are compared to several MC simulations and theoretical calculations. Some of the results are represented in Figure 2. These results agree with the previous ATLAS measurements and are in fair agreement with the SM predictions in a wide kinematic range. However, data distributions are softer than predicted for higher values of the mass of the $t \bar{t}$ system and of the top quark transverse momentum, particularly in the case of the ALPGEN+HERWIG and POWHEG+PYTHIA generators. The distributions show some preference for HERAPDF1.5 when used in conjunction with a fixed-order NLO QCD calculation. More precise conclusions about PDFs will be possible from the comparison of these measurements to future calculations at NNLO+NNLL in QCD and after including electroweak effects.

Although there is some modulation, the results obtained in both differential cross-sections studies are consistent with the SM predictions, and will contribute to the tuning of the MC simulation.

\subsection{The single top production cross-section}

The single top cross-section has been measured by the ATLAS experiment in all three channels, using data from proton-proton collisions both at $7 \mathrm{TeV}$ and $8 \mathrm{TeV}$, corresponding to an integrated luminosity of $4.6 \mathrm{fb}^{-1}$ and $20.3 \mathrm{fb}^{-1}$, respectively.

Already at center-of-mass energy of $7 \mathrm{TeV}$, the cross-section of the $t$-channel of the single top quark production was measured. Here, separate measurements of the cross-section for the top quark $(t)$ and the anti-top quark $(\bar{t})$ were made and subsequently combined into an inclusive $t q+\bar{t} q$ cross-section [19]. The cross-sections were measured by performing a binned maximumlikelihood fit to the output distributions of neural networks. The resulting measurements are: $\sigma_{t q}=$ 46. \pm 1 (stat.) \pm 6 (syst.) pb , $\sigma_{\bar{t} q}=23 \pm 1$ (stat.) \pm 3 (syst.) pb, $\sigma_{(t q+\bar{t} q)}=68 \pm 8 \mathrm{pb}$, which are in 


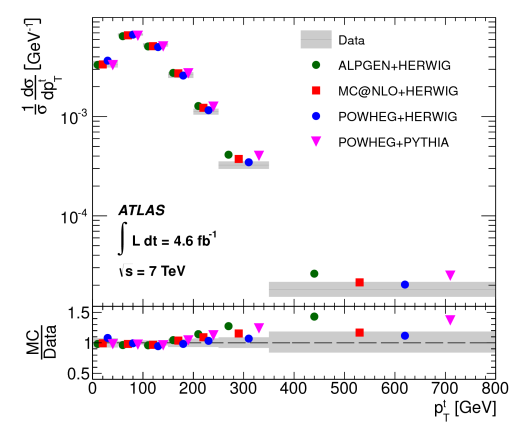

(a)

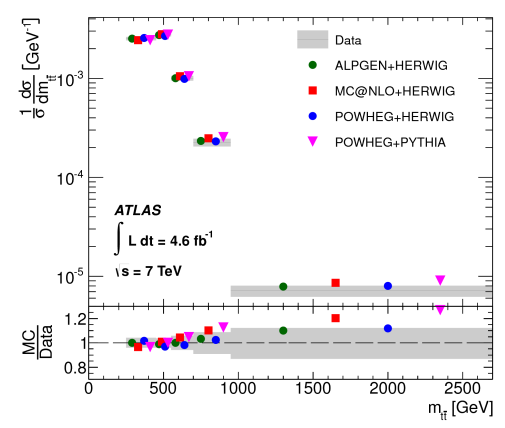

(b)

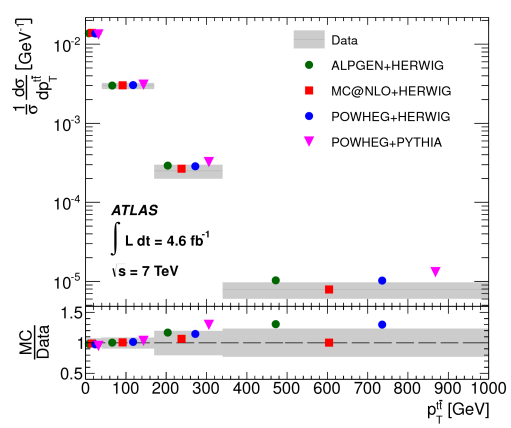

(c)

Figure 2: Normalised differential cross-sections for the (a) transverse momentum of the hadronically decaying top quark $\left(p_{\mathrm{T}}^{t}\right)$, and the (b) mass $\left(m_{t \bar{t}}\right)$ and the (c) transverse momentum $\left(p_{\mathrm{T}}^{t \bar{t}}\right)$ of the $t \bar{t}$ system. For more detailed information see [17].

excellent agreement with the SM expectation. The uncertainty on the combined measured crosssection is $\sim 12 \%$ and is dominated by systematic uncertainties. Using the ratio of the measured inclusive cross-section to its theoretical prediction, and assuming that $\left|V_{t b}\right|>>\left|V_{t s}\right|,\left|V_{t d}\right|$, the $V_{t b}$ coupling strength was determine as $\left|V_{t b}\right|=1.02 \pm 0.07$.

The single top quark $t$-channel analysis using $\sqrt{s}=8 \mathrm{TeV}$ data was performed in the single lepton channel and a fiducial cross-section was measured using neural-network discriminants [20]. The $t$-channel production cross-section in the fiducial volume close to the experimental acceptance was obtained from a binned maximum-likelihood fit to the neural-network discriminant: $\sigma_{\text {fid }}=3.37 \pm 0.05$ (stat.) \pm 0.47 (syst.) \pm 0.09 (lumi.) pb. The total inclusive $t$-channel cross-section can be extrapolated using the acceptance of the fiducial volume predicted by various MC generators. Using the acceptance from the MC@NLO+Herwig event generator, the inclusive crosssection obtained was: $\sigma_{\mathrm{t}-\text { chan }}=82.6 \pm 1.2$ (stat.) \pm 11.4 (syst.) \pm 3.1 (PDF) \pm 2.3 (lumi.) pb, which is consistent with the SM prediction. The $V_{t b}$ coupling strength can be determined from the ratio of the measured inclusive cross-section to the predicted cross-section. However, in order for its extrapolation, it is necessary to assume that the CKM matrix elements related to the top quark obey $\left|V_{t b}\right|>>\left|V_{t s}\right|,\left|V_{t d}\right|$. The coupling strength at the $W t b$ vertex was thus determined to be $\left|V_{t b}\right|=0.97_{-0.10}^{+0.09}$ and assuming that $\left|V_{t b}\right| \approx 1$ a lower limit was also obtained: $\left|V_{t b}\right|>0.78$ at $95 \%$ confidence level.

The $W t$-channel cross-section was obtained both at $7 \mathrm{TeV}$ and $8 \mathrm{TeV}$ in two different studies based on similar analysis methods (c.f. [21] and [22] respectively). In order to discriminate between the single top quark $W t$ signal events and the dominant background from top quark pairs, an analysis based on Boosted Decision Trees was used. A template fit to the final classifier distributions in several jet multiplicity events was then used to measure the signal cross-section. The $W t$-channel cross-section results are: $\sigma_{W t \text {-chan }}(7 \mathrm{TeV})=16.8 \pm 2.9$ (stat.) \pm 4.9 (syst.) pb [21], and $\sigma_{W t-\text { chan }}(8 \mathrm{TeV})=27.2 \pm 2.8$ (stat.) \pm 5.4 (syst.) pb [22] which are in good agreement with the SM expectation and have uncertainties of $\sim 34 \%$ and $\sim 23 \%$ respectively. From these results information on the CKM matrix element can be extracted assuming that the $W t$ production through $\left|V_{t s}\right|$ and $\left|V_{t d}\right|$ is small: at $7 \mathrm{TeV}$ the obtained coupling value was $\left|V_{t b}\right|=1.03_{-0.19}^{+0.16}$ and at $8 \mathrm{TeV}$ a 

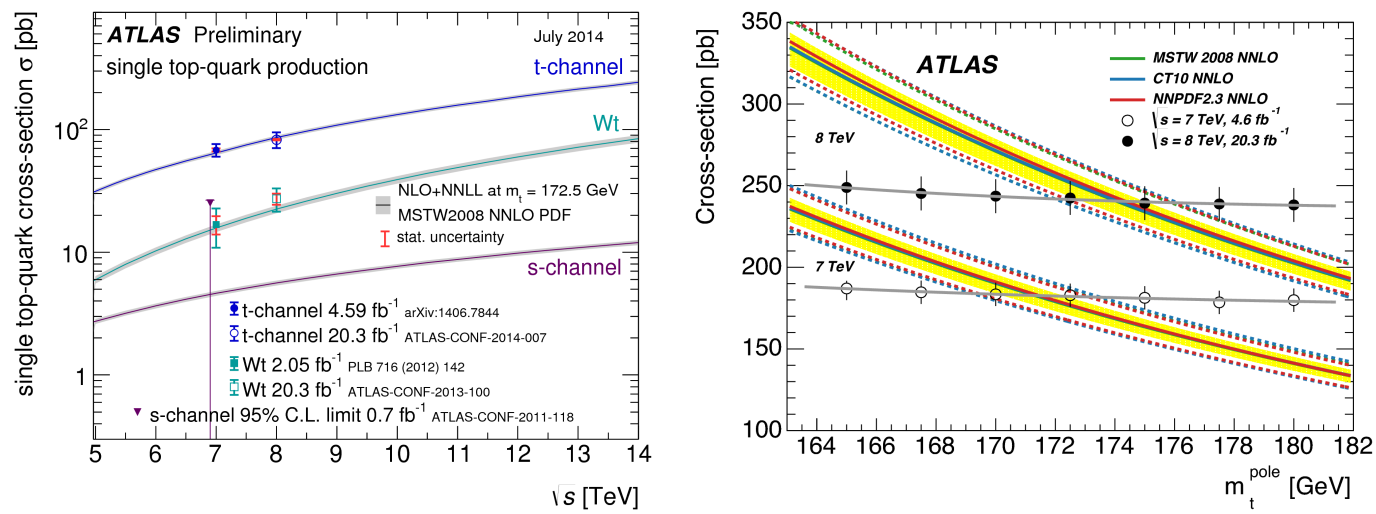

Figure 3: Left: Summary of measurements of the single top production cross-sections in various channels as a function of the center of mass energy compared to a theoretical calculation based on NLO QCD complemented with NNLL resummation [18]. For the $s$-channel only an upper limit is shown. Right: Predicted NNLO+NNLL ttbar production cross-sections at 7 and $8 \mathrm{TeV}$ as a function of $m_{t}^{\text {pole }}$, showing the central values and total uncertainty bands with several PDF sets. The yellow band shows the QCD scale uncertainty. The measurements of $\sigma_{t t b a r}$ are also shown, with their dependence on the assumed value of $m_{t}$ through acceptance and background corrections parametrised using $\sigma_{t \bar{t}}^{\text {theo }}\left(m_{t}^{\text {ref }}\right)$ referred below [15].

lower limit was obtained $\left|V_{t b}\right|>0.72 @ 95 \%$ CL.

A summary of the ATLAS results on the single top quark cross-sections for the various channels can be seen in Figure 3 as a function of the center of mass energy and compared to theoretical calculations based on NLO QCD complemented with NNLL resummation. For the $s$-channel only an upper limit is shown. Once again everything is in agreement with the SM expectations.

\section{Top Quark Mass}

The precise measurement of the top quark mass $m_{t}$ provides important consistency tests of the Standard Model. One can determine the mass of the top quark through direct reconstruction of the top decay products or extrapolate the pole mass $m_{t}^{\text {pole }}$ from the cross-section measurements, corresponding to the definition of the mass of a free particle. It is therefore interesting to compare the values of $m_{t}$ determined from the two approaches.

The $t \bar{t}$ dileptonic analysis [15] was used to determine the $m_{t}^{\text {pole }}$. The dependence of the crosssection predictions on the top quark pole mass are represented on Figure 3 (right) and were fitted with $\sigma_{t \bar{t}}^{\text {theo }}\left(m_{t}^{\text {ref }}\right)=\sigma\left(m_{t}^{\text {ref }}\right)\left(\frac{m_{t}^{\text {ref }}}{m_{t}^{\text {pole }}}\right)^{4}\left(1+a_{1} x+a_{2} x^{2}\right)$, where $m_{t}^{\text {ref }}=172.5 \mathrm{GeV}, x=\frac{\left(m_{t}^{\text {pole }}-m_{t}^{\text {ref }}\right)}{m_{t}^{\text {ref }}}$, and $\sigma\left(m_{t}^{r e f}\right), a_{1}$ and $a_{2}$ are free parameters. From the comparison of the theoretical and experimental curves on Figure 3 (right) an extraction of the top quark pole mass can be done by maximising a Bayesian likelihood as a function of $m_{t}^{\text {pole }}$. The combined result for 7 and $8 \mathrm{TeV}$ was obtained with $1.5 \%$ uncertainty as: $m_{t}^{\text {pole }}=172.9_{-2.6}^{+2.5} \mathrm{GeV}$. This can be directly compared with the world combination of the top quark mass obtained directly from its decay products $m_{t o p}=173.34 \pm$ 0.27 (stat.) \pm 0.71 (syst.) $\mathrm{GeV}$, which is the most precise measurement of the top mass so far, with total relative uncertainty of $0.4 \%$, dominated by the systematic uncertainties [3]. 


\section{Polarisation and Spin Correlations}

At the LHC, $t \bar{t}$ pairs are produced essentially unpolarised according to the SM. However, the spins of the top and the anti-top quark are expected to be correlated. New physics models beyond the SM might change the spin correlation of the $t$ and $\bar{t}$ by changing the spin of their daughter particles, or by changing the production mechanism of the $t \bar{t}$ pair.

The spin correlation and top polarisation can be extracted by analysing the angular distributions of the top quark decay products on $t \bar{t}$ events. The double differential distribution of the decay width, $\frac{1}{\sigma} \frac{d^{2} \sigma}{\mathrm{d} \cos \left(\theta_{+}\right) \mathrm{d} \cos \left(\theta_{-}\right)}=\frac{1}{4}\left(1+\alpha_{+} P_{+} \cos \left(\theta_{+}\right)+\alpha_{-} P_{-} \cos \left(\theta_{-}\right)+A \alpha_{+} \alpha_{-} \cos \left(\theta_{+}\right) \cos \left(\theta_{-}\right)\right)$ is proportional to the cosine of the angle, $\theta_{ \pm}$, between the momentum of the positively (negatively) charged lepton from the top quark (anti-top quark) decay and the top quark (anti-top quark) spin quantisation axis in the top quark (anti-top quark) rest frame. $\alpha$ represents the spin analysing power of the decay products, $P_{ \pm}$is the polarisation of the top/anti-top quark and $A$ is the asymmetry built from the ratio between the number of events where the top quark and anti-top quark spins are aligned $\left(N_{\Uparrow \Uparrow}\right.$ and $\left.N_{\Downarrow \Downarrow}\right)$ and the number of events where they are anti-alligned $\left(N_{\Uparrow \Downarrow}\right.$ and $\left.N_{\Downarrow \Uparrow}\right)$, $A=\frac{N_{\Uparrow \Uparrow}+N_{\Downarrow \Downarrow}-N_{\Uparrow \Downarrow}-N_{\Downarrow \Uparrow}}{N_{\Uparrow \Uparrow}+N_{\Downarrow \Downarrow}+N_{\Uparrow \Downarrow}+N_{\Downarrow \Uparrow}}$.

\subsection{Top quark polarisation}

The measurement of the top quark polarisation in $t \bar{t}$ events was performed in the single lepton and dilepton channels at $\sqrt{s}=7 \mathrm{TeV}$ [23]. The spin polarisations where determinded by fitting the angular distributions of the top decay produts with MC templates with assumed polarisations of $\alpha_{l} P= \pm 0.3$. Two extreme scenarios were considered in the production mechanisms of $t \bar{t}$ : the CP conservation (CPC) as in the SM and the maximally CP violating (CPV). For the CP conserving case, the top and anti-top quarks have the same $\alpha_{l} P$, whereas for the $\mathrm{CP}$ violating case, the top quark has opposite $\alpha_{l} P$ to the anti-top quark. From the binned likelihood fit to the angular data distributions, the following results were obtained for the two production mechanisms: $\alpha_{l} C_{C} P C=$ $-0.035 \pm 0.014$ (stat.) \pm 0.037 (syst.) and $\alpha_{l} C_{C} P V=-0.020 \pm 0.016$ (stat.) ${ }_{-0.017}^{0.013}$ (syst.). No deviation from the SM prediction were measured.

\subsection{Top quark spin correlation}

In order to extract the spin correlation strength from the selected data, four different observables are investigated [24], which are different linear combinations of components of the spin density matrix of $t \bar{t}$ production: the azimuthal difference $\Delta \Phi$ of the charged lepton momentum directions in the laboratory frame, the ratio of the squares of matrix elements for top quark pair production and decay from the fusion of like-helicity gluons with and without spin correlation at leading order (S-Ratio), and the product of $\cos \left(\theta_{+}\right)$and $\cos \left(\theta_{-}\right)$in both the helicity basis and the so called maximal basis, which maximizes the value of the spin correlation strength. A template fit is then performed for all four observables using templates with SM spin correlation and without it, both generated with MC@NLO. The result is expressed in the parameter $f_{S M}$ which is 1 for the SM predicted spin correlation case and $f_{S M}=0$ in the uncorrelated case. The $f_{S M}$ coeficient can be seen in Figure 4 (left) for all four observables. In order to obtain the measured spin correlation, the $f_{S M}$ has to be multiplied by the SM prediction. The spin correlation observables are in fact sensitive 


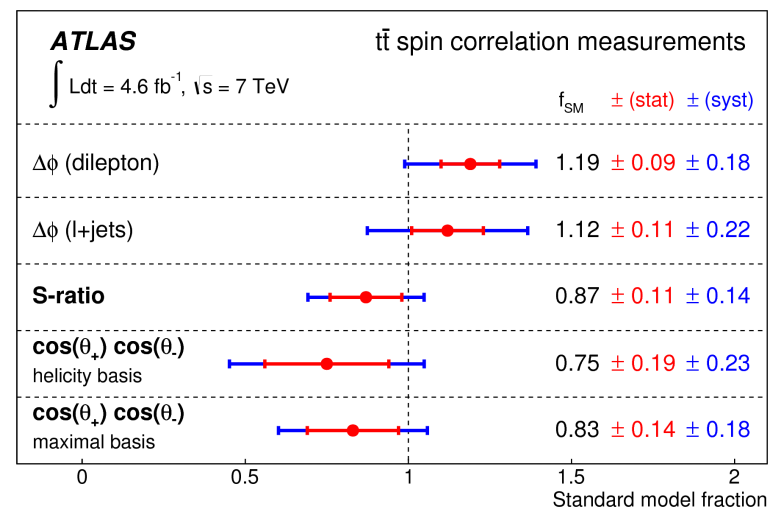

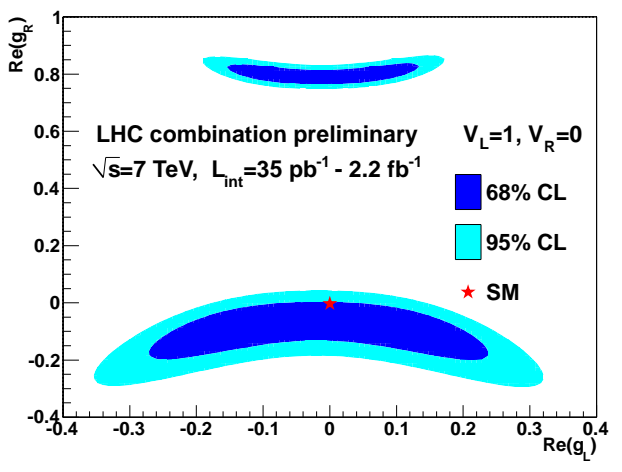

Figure 4: Left: Summary of the measurements of the fraction of $t$ and $\bar{t}$ events corresponding to the SM spin correlation hypothesis, $f_{S M}$, in the dilepton final stateand in the single-lepton final state. Dashed vertical line at $f_{S M}=1$ indicates the SM prediction [24]. Right: Allowed regions, at $68 \%$ and $95 \% \mathrm{CL}$, on the anomalous coupling plane $\left(g_{\mathrm{L}}, g_{\mathrm{R}}\right)$, for $V_{\mathrm{R}}=0$ and $V_{\mathrm{L}}=1[25]$.

to different properties of the production mechanism, being the $\Delta \Phi$ the most sensitive measurement, which as the advantage of not requiring the reconstruction of the $t \bar{t}$ system.

\section{5. $W$ boson polarisation in $t \rightarrow b W$ decays}

In the $\mathrm{SM}$, the $\mathrm{Wtb}$ vertex has a $(V-A)$ structure where, $\mathrm{V}$ and $\mathrm{A}$, are the vector and axialvector contributions to the vertex. The $\mathrm{W}$ bosons produced in these decays can have longitudinal, left-handed or right-handed polarisations with fractions $F_{0}, F_{\mathrm{L}}$ and $F_{\mathrm{R}}$, respectively equal to $0.687 \pm$ $0.005,0.311 \pm 0.005$ and $0.0017 \pm 0.0001$ at NNLO [26], in the SM. These fractions can be obtain from measurements of the angular distribution of the decay products of the $t$, in particular by reconstructing the angle $\theta_{l}^{*}$ defined as the angle between the momentum direction of the charged lepton from the decay of the $W$ boson and the momentum of the $W$ boson in the top quark rest frame. Here two methods were used: measuring the helicity fractions directly from a fit to the angular distribution or alternatively, using angular asymmetries $\left(A_{+}, A_{-}\right.$and $\left.A_{F B}\right)$ [27]. The combined results obtained by the ATLAS experiment are, $F_{0}=0.67 \pm 0.03$ (stat) \pm 0.06 (syst), $F_{\mathrm{L}}$ $=0.32 \pm 0.02$ (stat) \pm 0.03 (syst) and $F_{\mathrm{R}}=0.01 \pm 0.01$ (stat) \pm 0.04 (syst) [28], which agree with SM expectations. The measured fractions were used to probe the existence of possible anomalous $W t b$ couplings. Exclusion limits on the real components of the anomalous couplings $g_{\mathrm{L}}$ and $g_{\mathrm{R}}$ were set at $68 \%$ and $95 \%$ CL (Figure 4 right part [25]).

\section{Top Quark Electric Charge}

In order to confirm the SM prediction for the electric charge of the top quark and exclude the exotic model of $Q_{t}=-4 / 3|e|$, an analysis based on the product of the charges of the lepton and of the $b$-quark was made, resulting in a measured top charge of $Q_{t}=0.64 \pm 0.02$ (stat.) 0.08 (syst.) [29]. This is compatible with the SM expectation and the exotic model is now excluded by more than 8 standard deviations. 


\section{Charge Asymmetry}

The $t \bar{t}$ production is predicted by the SM to be symmetric at leading order (LO) under charge conjugation. However, at NLO for the $q \bar{q}$ and $q g$ production modes, there is a small preference to produce the $t(\bar{t})$ in the direction of the incoming quark (anti-quark). Thus, it is useful to define the charge asymmetry: $A_{C}^{t \bar{t}}=\frac{N(\Delta|y|>0)-N(\Delta|y|<0)}{N(\Delta|y|>0)+N(\Delta|y|<0)}$ where $\Delta|y|=\left|y_{t}\right|-\left|y_{\bar{t}}\right|$, with $y$ the rapidity of $t$ or $\bar{t}$, which at NLO in the SM is expected to be $A_{C}^{S M}=0.0123 \pm 0.0005$ for $7 \mathrm{TeV}$. In the single lepton channels, ATLAS measured the charge asymmetry as $A_{C}^{t \bar{t}}=0.006 \pm 0.010$ (stat.) \pm 0.005 (syst.) [30]. Furthermore, a combination [31] with the results of the CMS experiment has been done resulting in $A_{C}^{t \bar{t}}=0.005 \pm 0.007$ (stat.) \pm 0.006 (syst.).

\section{Top quark couplings to gauge bosons}

The precise measurements of the top quark couplings to the gauge boson $(\gamma, \mathrm{Z}, \mathrm{W}$ and $\mathrm{H})$ is of the utmost importance. Deviations from the SM predictions may indicate many sources of new physics. Several dedicated analyses were developed, both in the single lepton and dilepton channels of associate pair production with a gauge boson. These are particularly challenging channels which should be improved as more data is collected over time.

The $t \bar{t} \gamma$ production [32] was analysed by fitting the data with templates for prompt photons (well isolated) and hadron fakes, obtaining a cross-section value of $\sigma(t \bar{t} \gamma)=2.0 \pm 0.5$ (stat) \pm 0.7 (syst) \pm 0.08 (lumi.) pb, for events with photons with $p_{\mathrm{T}}>8 \mathrm{GeV}$ for which the expected SM cross section is $2.1 \pm 0.4 \mathrm{pb}$ (at $7 \mathrm{TeV}$ ).

For the $t \bar{t} V$ with $V=Z, W$ a combined dilepton (same-sign and opposite-sign) and trilepton result with $\sqrt{s}=8 \mathrm{TeV}$ was obtained simultaneously for both gauge bosons as: $\sigma_{t \bar{t} Z}=150_{-54}^{+58} \mathrm{fb}$ and $\sigma_{t \bar{t} W}=300_{-110}^{+140} \mathrm{fb}$, which are in agreement with the SM prediction [33].

The ATLAS experiment searched for $t \bar{t} H$ production in two of the higgs boson decay channels, $H \rightarrow b \bar{b}$ [34] and $H \rightarrow \gamma \gamma$ [35] both in single lepton and dilepton analysis. As no significant excess of events above the background expectation is found, combined $t \bar{t} H$ observed and expected 95\% confidence-level limits were set on the cross-section. By performing a fit allowing for an arbitrary Higgs boson signal strength, the ratio of the measured signal strength to the Standard Model expectation is found to be $\mu=1.6_{-1.1}^{+1.3}$.

\section{Conclusions}

The top quark studies with the ATLAS detector are well under way, now addressing precision measurements. Although no new physics was seen so far in the top quark sector, the performance of the LHC and the ATLAS experiment have been remarkable.

The work of S.Amor dos Santos was supported by Fundação para a Ciência e Tecnologia under project SFRH/BD/73438/2010.

\section{References}

[1] CDF Collaboration, Phys.Rev.Lett. 74 (1995) 2626-2631, [hep-ex/9503002] 
[2] D0 Collaboration, Phys.Rev.Lett. 74 (1995) 2632-2637, [hep-ex/9503003]

[3] ATLAS Collaboration, CDF Collaboration, CMS Collaboration, D0 Collaboration, arXiv:1403.4427[hep-ex], ATLAS-CONF-2014-008, CDF-NOTE-11071, CMS-PAS-TOP-13-014, D0-NOTE-6416 (2014).

[4] J. Beringer et al. (Particle Data Group), Phys. Rev. D 86, 010001 (2012)

[5] V. M. Abazov et al. [D0 Collaboration], Phys. Rev. D 85, 091104 (2012) [arXiv:1201.4156 [hep-ex]].

[6] M. Cacciari, M. Czakon, M. Mangano, A. Mitov, P. Nason, Phys.Lett. B710, 612-622 (2012).

[7] P. Bärnreuther, M. Czakon, A. Mitov, Phys. Rev. Lett. 109, 132001 (2012).

[8] M. Czakon, A. Mitov, JHEP 1212054 (2012), 10.1007/JHEP12(2012)054.

[9] M. Czakon, A. Mitov, JHEP 1301080 (2013), 10.1007/JHEP01(2013)080.

[10] M. Czakon, P. Fiedler, A. Mitov, Phys.Rev.Lett. 25110252004 (2013).

[11] N. Kidonakis, Phys. Rev. D 83, 091503 (2011) [arXiv:1103.2792 [hep-ph]].

[12] N. Kidonakis, Phys. Rev. D 82, 054018 (2010) [arXiv:1005.4451 [hep-ph]].

[13] N. Kidonakis, Phys. Rev. D 81, 054028 (2010) [arXiv:1001.5034 [hep-ph]].

[14] ATLAS Collaboration, JINST 3, S08003 (2008).

[15] ATLAS Collaboration, arXiv:1406.5375 [hep-ex], 2014, CERN-PH-EP-2014-124.

[16] ATLAS Collaboration, arXiv:1407.0891 [hep-ex], 2014, CERN-PH-EP-2014-114.

[17] ATLAS Collaboration, arXiv:1407.0371 [hep-ex], 2014, CERN-PH-EP-2014-099.

[18] ATLAS Collaboration, https://atlas.web.cern.ch/Atlas/GROUPS/PHYSICS/CombinedSummaryPlots/TOP/.

[19] ATLAS Collaboration, arXiv:1406.7844 [hep-ex], 2014, CERN-PH-EP-2014-133.

[20] ATLAS Collaboration, 2014, ATLAS-CONF-2014-007.

[21] ATLAS Collaboration, Physics Letters B 716 (2012) 142 - 159.

[22] ATLAS Collaboration, ATLAS-CONF-2013-100.

[23] ATLAS Collaboration, Phys. Rev. Lett. 111 (2013) 23, 232002,

[24] ATLAS Collaboration, arXiv:1407.4314 [hep-ex], (2014), CERN-PH-EP-2014-11.

[25] ATLAS Collaboration, ATLAS-CONF-2013-033, Mar 2013.

[26] A. Czarnecki, et al., Phys. Rev. D 81, 111503 (2010)

[27] J. A. Aguilar-Saavedra, et al., Eur. Phys. J. C 50, 519-533 (2007)

[28] ATLAS Collaboration, JHEP 1206, 088 (2012) [arXiv:1205.2484 [hep-ex]].

[29] ATLAS Collaboration, JHEP 1311, (2013), 031, arXiv:1307.4568 [hep-ex], CERN-PH-EP-2013-056,

[30] ATLAS Collaboration, JHEP 1402 (2014) 107, arXiv:1311.6724 [hep-ex], CERN-PH-EP-2013-177.

[31] CERN, Geneva, ATLAS-CONF-2014-012, Mar 2014.

[32] ATLAS Collaboration, ATLAS-CONF-2011-153.

[33] ATLAS Collaboration, ATLAS-CONF-2014-038, Jul 2014.

[34] ATLAS Collaboration, ATLAS-CONF-2014-011, Mar 2014.

[35] ATLAS Collaboration, ATLAS-CONF-2014-043", Jul 2014. 